\title{
THE DESIGN OF A NOVEL PRISMATIC DRIVE FOR A THREE-DOF PARALLEL-KINEMATICS MACHINE
}

\author{
J. Renotte, D. Chablat \\ Institut de Recherche en Communications \\ et Cybernétique de Nantes, UMR CNRS n 6597 \\ 1, rue de la Noë, 44321 Nantes, France \\ Damien.Chablat@irccyn.ec-nantes.fr
}

\author{
J. Angeles \\ Department of Mechanical Engineering \& \\ Centre for Intelligent Machines, McGill University \\ 817 Sherbrooke Street West, Montreal, Canada H3A 2K6 \\ angeles@cim.mcgill.ca
}

\section{ब্ঠি}

\section{BSTRACT}

The design of a novel prismatic drive is reported in this paper. This transmission is based on Slide-O-Cam, a cam mechanism with multiple rollers mounted on a common translating folFower. The design of Slide-O-Cam was reported elsewhere. This Arive thus provides pure-rolling motion, thereby reducing the friction of rack-and-pinions and linear drives. Such properties can be used to design new transmissions for parallel-kinematics 'machines. In this paper, this transmission is optimized to replace ball-screws in Orthoglide, a three-DOF parallel robot optimized 탕 machining applications.

\section{Introduction}

In robotics and mechatronics applications, whereby motion is controlled using a piece of software, the conversion of motion from rotational to translational is usually done by ball screws Oor linear actuators. Of these alternatives, ball screws are gainIng popularity, one of their drawbacks being the high number $\checkmark$ to moving parts that they comprise, for their functioning relies - n a number of balls rolling on grooves machined on a shaft; one more drawback of ball screws is their low load-carrying capacity, Istemming from the punctual form of contact by means of which foads are transmitted. Linear bearings solve these drawbacks to Some extent, for they can be fabricated with roller bearings, their drawback being that these devices rely on a form of direct-drive motor, which makes them expensive to produce and to maintain. A novel transmission, called Slide-O-Cam, was introduced in [1] (Fig. 1) to transform a rotation into a translation. Slide-O-Cam is composed of four major elements: (i) the frame, (ii) the cam, (iii) the follower and (iv) the rollers. The input axis on which the cam is mounted, the camshaft, is driven at a constant angular velocity. Power is transmitted to the output, the translating follower, which is the roller-carrying slider, by means of purerolling contact between cam and roller. The roller comprises two components, the pin and the bearing. The bearing is mounted at one end of the pin, while the other end is press-fit into the rollercarrying slider. Contact between cam and roller thus takes place at the outer surface of the bearing. The mechanism uses two conjugate cam-follower pairs, which alternately take over the motion transmission to ensure a positive action; rollers are driven by the cams, throughout a complete cycle. The main advantage of using a cam-follower mechanism instead of an alternative transmission to transform rotation into translation is that contact through a roller reduces friction, contact stress and wear.

This transmission will be optimized to replace the three ball screws used by the Orthoglide prototype [2]. Orthoglide features three prismatic joints mounted orthogonaly, three identical legs and a mobile platform, which moves in the Cartesian $x-y-z$ space with fixed orientation, as shown in Fig. 2. The motor used to move each axis is SANYO DENKI (ref. P30B08075D) with a constant torque of $1.2 \mathrm{Nm}$ from 0 to $3000 \mathrm{rpm}$. This property enables the mechanism to move throughout the workspace a $4 \mathrm{~kg}$ load with an acceleration of $17 \mathrm{~ms}^{-2}$ and a velocity of $1.3 \mathrm{~ms}^{-1}$. On the ball screws, the pitch is $50 \mathrm{~mm}$ per cam turn. The minimum radius of the camshaft is $8.5 \mathrm{~mm}$. Unlike Lampinen [3], who used a genetic algorithm, we use a deterministic method, while taking into account geometric and machining constraints as 


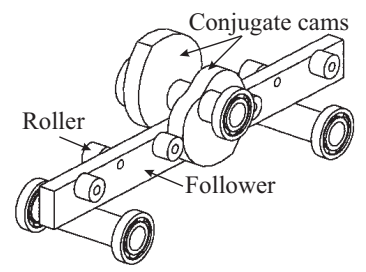

Figure 1. Layout of Slide-O-Cam

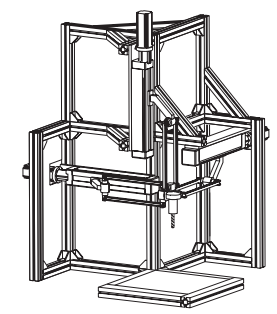

Figure 2. The Orthoglide

outlined in [7]. In section 2, we introduce the relations describing the cam profile and the mechanism kinematics. In Section 3, we derive conditions on the design parameters so as to have a fully convex cam profile, to avoid undercutting, and to have a geometrically feasible mechanism. In Section 4, the pressure angle, a key performance index of cam mechanisms, is studied in order to choose the design parameters that give the best pressure-angle distribution, a compromise being done with the accuracy of the mechanism.

\section{Synthesis of the Planar Cam Mechanism}

Let the $x-y$ frame be fixed to the machine and the $u$-v frame be attached to the cam, as depicted in Fig. 3. $O_{1}$ is the origin of both frames, while $\mathrm{O}_{2}$ is the center of the roller and $C$ is the contact point between cam and roller. The geometric parameters

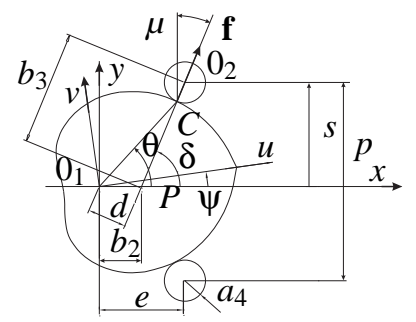

Figure 3. Parameterization of Slide-O-Cam

defining the cam mechanism are illustrated in the same figure. The notation of this figure is based on the general notation introduced in [5], namely, (i) $p$ : the pitch, i.e., the distance between the center of two rollers on the same side of the follower; (ii) $e$ : distance between the axis of the cam and the line of centers of the rollers; (iii) $a_{4}$ : radius of the roller bearing, i.e., the radius of the roller; (iv) $\psi$ : angle of rotation of the cam, the input of the mechanism; (v) $s$ : position of the center of the roller, i.e, the displacement of the follower, the output of the mechanism; (vi) $\mu$ : pressure angle; (vii) $\mathbf{f}$ : force transmitted from the cam to the roller. In this paper, $p$ is set to $50 \mathrm{~mm}$, in order to meet the Orthoglide specifications.

The above parameters as well as the contact surface on the cam, are determined by the geometric relations dictated by the Aronhold-Kennedy Theorem in the plane [6]. When the cam makes a complete turn $(\Delta \psi=2 \pi)$, the displacement of the roller is equal to $p$, the distance between two rollers on the same side of the roller-carrying slider $(\Delta s=p)$. Furthermore, if we consider the initial configuration of the roller as depicted in Fig. 4, the roller is on the lower side of the $x$-axis for $\psi=0$, so that we have $s(0)=-p / 2$. Hence, the input-output function $s$ is

$$
s(\psi)=\frac{p}{2 \pi} \psi-\frac{p}{2}
$$

The expression for the first and second derivatives of $s(\psi)$ with respect to $\psi$ will be needed:

$$
s^{\prime}(\psi)=p /(2 \pi) \quad \text { and } \quad s^{\prime \prime}(\psi)=0
$$

The cam profile is determined by the displacement of the contact point $C$ around the cam. The Cartesian coordinates of this point in the $u$-v frame take the form [5]

$$
\begin{aligned}
& u_{c}(\psi)=b_{2} \cos \psi+\left(b_{3}-a_{4}\right) \cos (\delta-\psi) \\
& v_{c}(\psi)=-b_{2} \sin \psi+\left(b_{3}-a_{4}\right) \sin (\delta-\psi)
\end{aligned}
$$

with coefficients $b_{2}, b_{3}$ and $\delta$ given by

$$
\begin{aligned}
b_{2} & =-s^{\prime}(\psi) \sin \alpha_{1} \\
b_{3} & =\sqrt{\left(e+s^{\prime}(\psi) \sin \alpha_{1}\right)^{2}+\left(s(\psi) \sin \alpha_{1}\right)^{2}} \\
\delta & =\arctan \left(\frac{-s(\psi) \sin \alpha_{1}}{e+s^{\prime}(\psi) \sin \alpha_{1}}\right)
\end{aligned}
$$

where $\alpha_{1}$ is the directed angle between the axis of the cam and the translating direction of the follower; $\alpha_{1}$ is positive in the ccw direction. Considering the orientation adopted for the input angle $\psi$ and for the output $s$, as depicted in Fig. B, we have

$$
\alpha_{1}=-\pi / 2
$$

We now introduce the nondimensional design parameter $\eta$, which will be extensively used:

$$
\eta=e / p
$$

Thus, from Eqs. (1), (2), (4a-c), (5) and (6), we compute the expressions for coefficients $b_{2}, b_{3}$ and $\delta$ as

$$
\begin{aligned}
b_{2} & =\frac{p}{2 \pi} \\
b_{3} & =\frac{p}{2 \pi} \sqrt{(2 \pi \eta-1)^{2}+(\psi-\pi)^{2}} \\
\delta & =\arctan \left(\frac{\psi-\pi}{2 \pi \eta-1}\right)
\end{aligned}
$$


whence a first constraint on $\eta, \eta \neq 1 /(2 \pi)$, is derived. An $e x$ tended angle $\Delta$ is introduced [7], so that the cam profile closes. Angle $\Delta$ is obtained as the root of the equation $v_{c}(\psi)=0$. In the case of Slide-O-Cam, $\Delta$ is negative, as shown in Fig. 5. Consequently, the cam profile closes within $\Delta \leq \psi \leq 2 \pi-\Delta$.

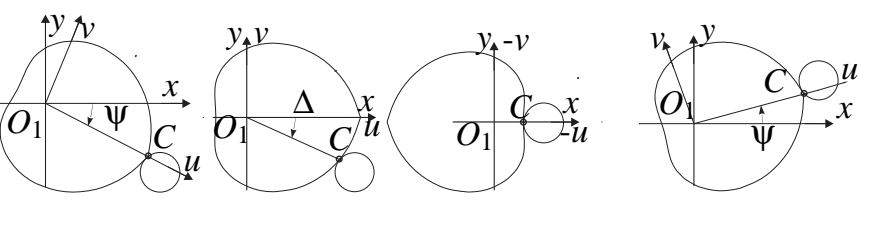
(a) $\psi=\Delta$
(b) $\psi=0$
(c) $\psi=\pi$
(d) $\psi=2 \pi-\Delta$

Figure 5. Extended angle $\Delta$

\subsection{Pitch-Curve Determination}

The pitch curve is the trajectory of the center $\mathrm{O}_{2}$ of the roller, distinct from the trajectory of the contact point $C$, which produces the cam profile. The Cartesian coordinates of point $\mathrm{O}_{2}$ in the $x-y$ frame are $(e, s)$, as depicted in Fig. 3. Hence, the Cartesian coordinates of the pitch-curve in the $u-v$ frame are

$$
\begin{aligned}
& u_{p}(\psi)=e \cos \psi+s(\psi) \sin \psi \\
& v_{p}(\psi)=-e \sin \psi+s(\psi) \cos \psi
\end{aligned}
$$

\subsection{Geometric Constraints on the Mechanism}

In order to lead to a feasible mechanism, the radius $a_{4}$ of the roller must satisfy two conditions, as shown in Fig. 6a:

- Two consecutive rollers on the same side of the roller-carrying slider must not be in contact. Since $p$ is the distance between the center of two consecutive rollers, we have the constraint $2 a_{4}<p$. Hence the first condition on $a_{4}$ :

$$
a_{4} / p<1 / 2
$$

- The radius $b$ of the shaft on which the cams are mounted must be taken into consideration. Hence, we have the constraint $a_{4}+$ $b \leq e$, the second constraint on $a_{4}$ in terms of the parameter $\eta$ thus being

$$
a_{4} / p \leq \eta-b / p
$$

Considering the initial configuration of the roller, as depicted in Fig. 4 , the $v$-component of the Cartesian coordinate of the contact point $C$ is negative in this configuration, i.e., $v_{c}(0) \leq 0$. Considering the expression for $v_{c}(\psi)$ and for parameters $b_{3}$ and $\delta$ given

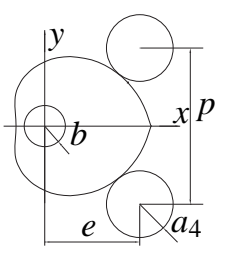

(a)

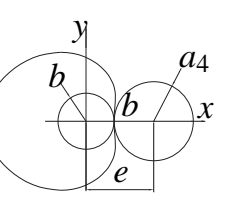

(b)
Figure 6. Constraints on the radius of the roller

in Eqs. (3b), (7b \& c), respectively, the above relation leads to the condition:

$$
\left(\frac{p}{2 \pi a_{4}} \sqrt{(2 \pi \eta-1)^{2}+(-\pi)^{2}}-1\right) \sin \left[\arctan \left(\frac{-\pi}{2 \pi \eta-1}\right)\right] \leq 0
$$

Further, we define $A$ and $B$ as:

$$
A=\frac{p}{2 \pi a_{4}} \sqrt{(2 \pi \eta-1)^{2}+\pi^{2}}-1 \text { and } B=\sin \left[\arctan \left(\frac{-\pi}{2 \pi \eta-1}\right)\right]
$$

Since $(2 \pi \eta-1)^{2}>0$, we have,

$$
\sqrt{(2 \pi \eta-1)^{2}+\pi^{2}}>\pi
$$

Hence, $A>p /\left(2 a_{4}\right)-1$. Furthermore, from the constraint on $a_{4}$, stated in Eq. (9), we have $p /\left(2 a_{4}\right)-1>0$, whence $A>0$. Consequently, the constraint $v_{c}(0) \leq 0$ leads to the constraint $B \leq$ 0 . We rewrite the expression for $B$, by using the trigonometric relation,

$$
B \leq 0 \Longleftrightarrow \frac{-\pi}{(2 \pi \eta-1) \sqrt{1+\pi^{2} /(2 \pi \eta-1)^{2}}} \leq 0
$$

which holds only if $2 \pi \eta-1>0$. Finally, the constraint $v_{c}(0) \leq 0$ leads to a constraint on $\eta$ :

$$
\eta>1 /(2 \pi)
$$

\subsection{Pressure Angle}

The pressure angle is defined as the angle between the common normal at the cam-roller contact point $C$ and the velocity of the follower [8], as depicted in Fig. 3, where the presure angle is denoted by $\mu$. This angle plays an important role in cam design. The smaller $|\mu|$, the better the force transmission. In the case of high-speed operations, i.e., angular velocities of cams exceeding $50 \mathrm{rpm}$, the recommended bounds of the pressure angle are within $30^{\circ}$. Nevertheless, as it is not always possible to have a 
pressure angle that remains below $30^{\circ}$, we adopt the service factor, which is the percentage of the working cycle with a pressure angle within $30^{\circ}$ [7]. The service angle will be useful to take into consideration these notions in the ensuing discussion, when optimizing the mechanism.

For the case at hand, the expression for the pressure angle $\mu$ is given in [8] as

$$
\mu=\arctan \left(\frac{s^{\prime}(\psi)-e}{s(\psi)}\right)
$$

Considering the expressions for $s$ and $s^{\prime}$, and using the parameter $\eta$ given in Eqs. (1), (2a) and (6), respectively, the expression for the pressure angle becomes

$$
\mu=\arctan \left(\frac{1-2 \pi \eta}{\psi-\pi}\right)
$$

We are only interested in the value of the pressure angle with the cam driving the roller, which happens with

$$
\pi \leq \psi \leq 2 \pi-\Delta
$$

Indeed, if we start the motion in the initial configuration depicted in Fig. 5b, with the cam rotating in the ccw direction, the cam begins to drive the roller only when $\psi=\pi$; and the cam can drive the follower until contact is lost, i.e., when $\psi=2 \pi-\Delta$, as shown in Figs. Fe \& d.

Nevertheless, as shown in Fig. 7, the conjugate cam can also drive the follower when $0 \leq \psi \leq \pi-\Delta$; there is therefore a common interval, for $\pi \leq \psi \leq \pi-\Delta$, during which two cams can drive the follower. In this interval, the conjugate cam can drive a roller with lower absolute values of the pressure angle. We assume that, when the two cams can drive the rollers, the cam with the lower absolute value of pressure angle effectively drives the follower. Consequently, we are only interested in the value of the pressure angle in the interval,

$$
\pi-\Delta \leq \psi \leq 2 \pi-\Delta
$$

We study here the influence of parameters $\eta$ and $a_{4}$ on the values of the pressure angle while the cam drives the roller, i.e., with $\pi-\Delta \leq \psi \leq 2 \pi-\Delta$, as explained above.

- Influence of parameter $\eta$ : Figure 8 shows the influence of the parameter $\eta$ on the pressure angle, with $a_{4}$ and $p$ being fixed. From these plots we have one result: The lower $\eta$, the lower $|\mu|$.

- Influence of the radius of the roller $a_{4}: a_{4}$ does not appear in the expression for the pressure angle, but it influences the value of the extended angle $\Delta$, and hence, the plot boundaries of the pressure angle, as shown in Fig. 7 .

By computing the value of the extended angle $\Delta$ for several values of $a_{4}$, we can say that the higher $a_{4}$, the lower $|\Delta|$. Consequently, since the boundaries to plot the pressure angle are $\pi-\Delta$ and $2 \pi-\Delta$, we can say that when we increase $a_{4},-\Delta$ decreases and the boundaries are translated toward the left, i.e., toward higher absolute values of the pressure angle.
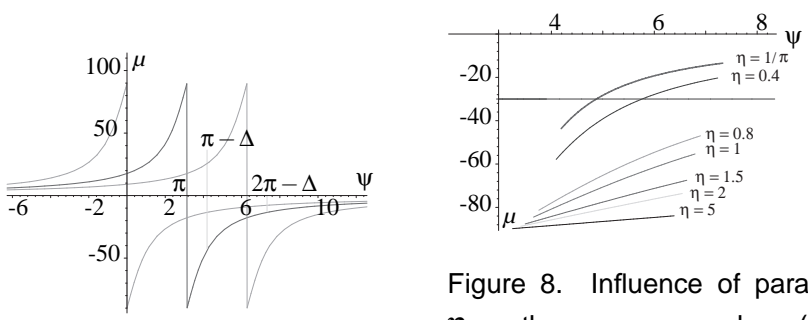

Figure 8. Influence of parameter $\eta$ on the pressure angle $\mu$ (in deFigure 7. Pressure angle distribu- gree), with $p=50 \mathrm{~mm}$ and $a_{4}=$ tion $10 \mathrm{~mm}$

\section{Convexity of the Cam Profile and Undercutting}

In order to enhance machining accuracy, we need the cam profile to be fully convex. In this section we establish conditions on the design parameters $\eta$ and $a_{4}$ in order to have a fully convex cam profile. So, we study the sign of the curvature of the cam profile via that of the pitch curve. Furthermore, for cam design in roller-follower mechanisms, we should also consider undercutting. Undercutting occurs when the radius of the roller is greater than or equal to the minimum absolute value of the radius of curvature of the pitch curve. Upon avoiding undercutting, the sign of the curvature of the pitch curve is identical to that of the cam profile.

\subsection{Curvature of the Cam Profile}

The curvature of any planar parametric curve, in terms of the Cartesian coordinates $u$ and $v$, and parameterized with any parameter $\psi$, is given by [8]:

$$
\kappa=\frac{v^{\prime}(\psi) u^{\prime \prime}(\psi)-u^{\prime}(\psi) v^{\prime \prime}(\psi)}{\left[u^{\prime}(\psi)^{2}+v^{\prime}(\psi)^{2}\right]^{3 / 2}}
$$

The sign of $\kappa$ in Eq. (16) tells whether the curve is convex or concave at a point: a positive $\kappa$ implies a convexity, while a negative $\kappa$ implies a concavity at that point. To obtain the curvature of the cam profile for a given roller-follower, we use the Cartesian coordinates of the pitch curve, since obtaining its first and second derivatives leads to simpler expressions as compared with those associated with the cam profile itself. Then, the curvature of the cam profile is derived by a simple geometric relationship between the curvatures of the pitch curve and of the cam profile. 
The Cartesian coordinates of the pitch curve were recalled in Eqs. (8a \& b), while Eqs. (2 a \& b) give their first and second derivatives

$$
s(\psi)=p /(2 \pi) \psi-p / 2, \quad s^{\prime}(\psi)=p /(2 \pi), \quad s^{\prime \prime}(\psi)=0
$$

With the above-mentioned expressions, we can compute the first and second derivatives of the Cartesian coordinates of the pitch curve with respect to the angle of rotation of the cam, $\psi$ :

$$
\begin{aligned}
u_{p}^{\prime}(\psi) & =\left(s^{\prime}(\psi)-e\right) \sin \psi+s(\psi) \cos \psi \\
v_{p}^{\prime}(\psi) & =\left(s^{\prime}(\psi)-e\right) \cos \psi-s(\psi) \sin \psi \\
u_{p}^{\prime \prime}(\psi) & =\left(2 s^{\prime}(\psi)-e\right) \cos \psi-s(\psi) \sin \psi \\
v_{p}^{\prime \prime}(\psi) & =-\left(2 s^{\prime}(\psi)-e\right) \sin \psi-s(\psi) \cos \psi
\end{aligned}
$$

By substituting $\eta, \eta=e / p$, along with Eqs. (17a-d), into Eq. (16), the curvature $\kappa_{p}$ of the pitch curve is obtained as

$$
\kappa_{p}=\frac{2 \pi}{p} \frac{\left[(\psi-\pi)^{2}+2(2 \pi \eta-1)(\pi \eta-1)\right]}{\left[(\psi-\pi)^{2}+(2 \pi \eta-1)^{2}\right]^{3 / 2}}
$$

provided that the denominator never vanishes for any value of $\psi$, i.e., provided that

$$
\eta \neq 1 /(2 \pi)
$$

Let $\rho_{c}$ and $\rho_{p}$ be the radii of curvature of both the cam profile and the pitch curve, respectively, and $\kappa_{c}$ the curvature of the cam profile. Since the curvature is the reciprocal of the radius of curvature, we have $\rho_{c}=1 / \kappa_{c}$ and $\rho_{p}=1 / \kappa_{p}$. Furthermore, due to the definition of the pitch curve, it is apparent that

$$
\rho_{p}=\rho_{c}+a_{4}
$$

Writing Eq. (20) in terms of $\kappa_{c}$ and $\kappa_{p}$, we obtain the curvature of the cam profile as

$$
\kappa_{c}=\frac{\kappa_{p}}{1-a_{4} \kappa_{p}}
$$

with $\kappa_{p}$ given in Eq. (18). As we saw previously, we want the cam profile to be fully convex, which happens if the pitch curve is fully convex too. We thus find first the convexity condition of the pitch curve.

\subsection{Convexity Condition of the Pitch Curve}

Considering the expression for $\kappa_{p}$ in Eq. (18), we have, for every value of $\psi, \kappa_{p} \geq 0$ if $(2 \pi \eta-1)(\pi \eta-1) \geq 0$ and $\eta \neq 1 /(2 \pi)$, whence the condition on $\eta$ :

$$
\kappa_{p} \geq 0 \text { if } \eta \in[0,1 /(2 \pi)[\cup[1 / \pi,+\infty[
$$

Figure 9 shows pitch curve profiles and their curvatures for two values of $\eta$. The condition on $\eta$ given in Eq. (22) must be

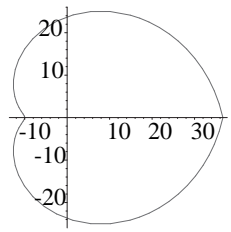

(a) $\eta=0.2(\eta \in] \frac{1}{2 \pi}, \frac{1}{\pi}[)$

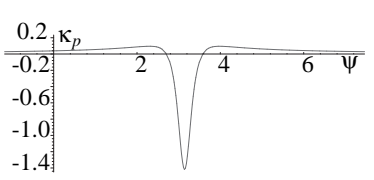

(c) Pitch curve curvature with $\eta=0.2(\eta \in] \frac{1}{2 \pi}, \frac{1}{\pi}[)$

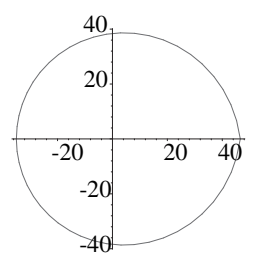

(b) $\eta=0.7(\eta>1 / \pi)$

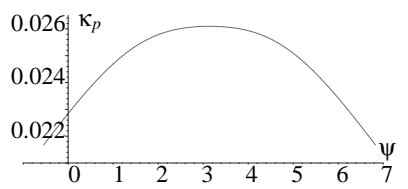

(d) Pitch curve curvature with $\eta=0.7(\eta>1 / \pi)$
Figure 9. Concave (a) and convex (b) pitch curve profiles and their corresponding curvatures with $p=50 \mathrm{~mm}$

combined with the condition appearing in Eq. (12), $\eta>1 /(2 \pi)$; hence, the final convexity condition of the pitch curve is:

$$
\eta \geq 1 / \pi
$$

\subsection{Undercutting Avoidance}

We assume in this subsection that the pitch curve is fully convex, i.e., $\kappa_{p} \geq 0$ and $\eta \geq 1 / \pi$. In order to avoid undercutting, i.e., in order to have both the cam profile and the pitch curve fully convex, we need $\kappa_{c}$ to be positive. Considering the expression for the curvature of the cam profile $\kappa_{c}$ of Eq. (21), the condition to avoid undercutting is $1-a_{4} \kappa_{p}>0$, whence the condition on the radius of the follower $a_{4}$ is

$$
a_{4}<\frac{1}{\kappa_{p}(\psi)} \quad \forall \psi \in \mathbb{R}
$$

Since $\kappa_{p}$ is positive, this condition can be written as

$$
a_{4}<\frac{1}{\kappa_{p \max }} \quad \text { with } \quad \kappa_{p \max }=\max _{\psi \in \mathbb{R}} \kappa_{p}(\psi)
$$


- Expression for $\kappa_{p \max }$ : In order to compute the expression for $\kappa_{p \max }$, we need the first derivative $\kappa_{p}^{\prime}$ of $\kappa_{p}$ with respect to $\psi$ and its roots. With the condition $\eta \geq 1 / \pi$, the expression for $\kappa_{p}$ given in Eq. (18) is differentiable for every value of $\psi$. Thus, we obtain

$$
\kappa_{p}^{\prime}=-\frac{2 \pi}{p} \frac{(\psi-\pi)\left(\psi^{2}-2 \pi \psi+\pi^{2}+4 \eta^{2} \pi^{2}-10 \eta \pi+4\right)}{\left[(\psi-\pi)^{2}+(2 \pi \eta-1)^{2}\right]^{5 / 2}}
$$

The roots of $\kappa_{p}^{\prime}$ are, apparently, $\psi_{1}=\pi$ and the roots $\psi_{2}$ and $\psi_{3}$ of the polynomial

$$
P(\psi)=\psi^{2}-2 \pi \psi+\pi^{2}+4 \eta^{2} \pi^{2}-10 \eta \pi+4
$$

Let $\beta_{\psi}$ be the discriminant of the equation $P=0$, i.e.,

$$
\beta_{\psi}=-4 \eta^{2} \pi^{2}+10 \eta \pi-4
$$

Therefore, the sign of $\beta_{\psi}$ and, consequently, the roots $\psi_{2}$ and $\psi_{3}$, depend on the value of $\eta$. Let $\beta_{\eta}$ be the discriminant of $\beta_{\psi}=0$, a quadratic equation in $\eta$. Hence, $\beta_{\eta}=9 \pi^{2}$, which is positive. The two roots of $\beta_{\psi}$ are $1 / 2 \pi$ and $2 / \pi$. Thus,

$$
\begin{aligned}
& \beta_{\psi}>0 \quad \text { if } \quad \eta \in\left[\frac{1}{\pi}, \frac{2}{\pi}\left[\text { or } \beta_{\psi}<0 \text { if } \eta \in\right] \frac{2}{\pi},+\infty[\right. \\
& \beta_{\psi}=0 \text { if } \eta=\frac{2}{\pi}
\end{aligned}
$$

We now study the roots of $\kappa_{p}^{\prime}$ according to the value of $\eta$.

- $\eta \in\left[1 / \pi, 2 / \pi\left[: \beta_{\psi}>0\right.\right.$, and the polynomial $P$ has two roots $\psi_{2}$ and $\psi_{3}$, so that $\kappa_{p}^{\prime}$ has three roots:

$$
\begin{aligned}
& \psi_{1}=\pi \\
& \psi_{2}=\pi+\sqrt{-4 \eta^{2} \pi^{2}+10 \eta \pi-4} \\
& \psi_{3}=\pi-\sqrt{-4 \eta^{2} \pi^{2}+10 \eta \pi-4}
\end{aligned}
$$

- $\eta \in] 2 / \pi,+\infty\left[: \beta_{\psi}<0\right.$, and the polynomial $P$ has no real roots, so that $\kappa_{p}^{\prime}$ has only one root, $\psi_{1}=\pi$.

- $\eta=2 / \pi: \beta_{\psi}=0$, and the polynomial $P$ has one double root equal to $\pi$, so that $\kappa_{p}^{\prime}$ has one triple root $\psi_{1}=\pi$.

To decide whether these roots correspond to minima or maxima, we need to know the sign of the second derivative $\kappa_{p}^{\prime \prime}$ of $\kappa_{p}$ with respect to $\psi$, for the corresponding values of $\psi$. If the second derivative is negative, the stationary value is a maximum; if positive, a minimum. The expressions for the second derivatives were computed with Maple, for the values of $\psi$ given in

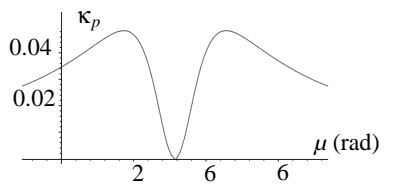

(a) $\eta=1 / \pi$

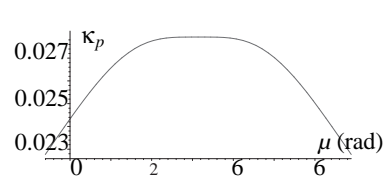

(b) $\eta=2 / \pi$
Figure 10. Pitch-curve curvature for $p=50 \mathrm{~mm}$ Eqs. (26a-c):

$$
\begin{aligned}
\kappa_{p}^{\prime \prime}\left(\psi_{1}\right) & =\frac{4 \pi(\eta \pi-2)}{p|2 \eta \pi-1|^{3}(2 \eta \pi-1)} \\
\kappa_{p}^{\prime \prime}\left(\psi_{2}\right)=\kappa_{p}^{\prime \prime}\left(\psi_{3}\right) & =\frac{8 \pi(\eta \pi-2)}{9 p(2 \eta \pi-1) \sqrt{6 \eta \pi-3}}
\end{aligned}
$$

- If $\eta \in\left[1 / \pi, 2 / \pi\left[, \kappa_{p}^{\prime \prime}\left(\psi_{1}\right)>0\right.\right.$ and $\kappa_{p}^{\prime \prime}\left(\psi_{2}\right)=\kappa_{p}^{\prime \prime}\left(\psi_{3}\right)<0$, the curvature of the pitch curve has one local minimum for $\psi_{1}$ and two maxima, for $\psi_{2}$ and $\psi_{3}$. Hence, the value of $\kappa_{p \max }$ is

$$
\kappa_{p \max 1}=\kappa_{p}\left(\psi_{2}\right)=\kappa_{p}\left(\psi_{3}\right)=\frac{4 \pi}{3 p \sqrt{6 \eta \pi-3}}
$$

Figure 10a shows a plot of the pitch-curve curvature with $\eta=$ $1 / \pi$ and $p=50 \mathrm{~mm}$. Since $\eta$ is taken equal to the convexity limit $1 / \pi$, the curvature remains positive and only vanishes for $\psi=\pi$.

- If $\eta \in] 2 / \pi,+\infty\left[, \kappa_{p}^{\prime \prime}\left(\psi_{1}\right)<0\right.$, the curvature of the pitch curve has a maximum for $\psi_{1}$. Hence, the value of $\kappa_{p \max }$ is

$$
\kappa_{p \max 2}=\kappa_{p}\left(\psi_{1}\right)=\frac{4 \pi}{p} \frac{\left(2 \eta^{2} \pi^{2}-3 \eta \pi+1\right)}{\left(4 \eta^{2} \pi^{2}-4 \eta \pi+1\right)^{3 / 2}}
$$

Figure 9 d shows a plot of the pitch-curve curvature with $\eta=0.7$ $(\eta>2 / \pi)$ and $p=50 \mathrm{~mm}$.

- If $\eta=2 / \pi, \kappa_{p}^{\prime \prime}\left(\psi_{1}\right)=0$, we cannot tell whether we are in the presence of a maximum or a minimum. We solve this uncertainty graphically, by plotting the curvature of the pitch curve for $\eta=2 / \pi$ and $p=50 \mathrm{~mm}$. Figure $10 \mathrm{~b}$ reveals that the curvature has a maximum for $\psi_{1}$. The value of this maximum can be obtained by substituting $\eta$ by $2 / \pi$ into either $\kappa_{p \max 1}$ or $\kappa_{p \max 2}$, expressed in Eqs. (27) and (28), respectively.

In summary, to have a fully convex cam profile, taking the geometric constraints on the mechanism into consideration, parameter $\eta$ must obey the condition given in Eq. (23), i.e. $\eta \geq 1 / \pi$. We combine the condition on $a_{4}$ to avoid undercutting, as given in Eq. (24), with the geometric constraints on the mechanism, as given in Eqs. (9) and (10), which are, respectively, $a_{4} / p<1 / 2$ and $a_{4} / p \leq \eta-b / p$ :

$$
\text { if } \eta \in\left[\frac{1}{\pi}, \frac{2}{\pi}\right], a_{4}<\min \left\{\frac{1}{\kappa_{p \max 1}}, \frac{p}{2}, \eta p-b\right\}
$$




$$
\text { if } \eta \in\left[\frac{2}{\pi},+\infty\left[, a_{4}<\min \left\{\frac{1}{\kappa_{p \max 2}}, \frac{p}{2}, \eta p-b\right\}\right.\right.
$$

where $\kappa_{p \max 1}$ and $\kappa_{p \max 2}$ are given in Eqs. 27) and (28), respectively.

\section{Optimization of the Roller Pin}

We concluded in previous section that the lowest values of parameters $\eta$ and $a_{4}$ led to the lowest values of the pressure angle. Nevertheless, we must take into consideration that the smaller the radius of the roller, the bigger the deformation of the roller pin, and hence, a decrease of the stiffness and the accuracy of the mechanism. In this section we formulate and solve an optimization problem to find the best compromise on parameters $\eta$ and $a_{4}$ to obtain the lowest pressure angle values with an acceptable deformation of the roller pin.

\subsection{Minimization of the Elastic Deformation on the Roller Pins}

Here we find the expression for the maximum elastic deformation on the pin, which will be minimized under given constraints. Figure 11 displays the free part of the pin, i.e., the part not fixed to the roller-carrying slider, as a cantilever beam, where the load $F=\sqrt{f_{x}^{2}+f_{y}^{2}}$ denotes the magnitude of the force $\mathbf{f}$ transmitted by the cam. This force is applied at a single point at the end of the pin in the worst loading case. Although the dimensions of the pin are not those of a simple beam, we assume below that the pin can be modelled as such, in order to obtain an explicit formula for its deflection. This assumption was found to be plausible by testing it with FEA [9].

$\begin{array}{lll}y \quad & \mathbf{f} \downarrow \mathbf{f} \backslash y \\ & L & \end{array}$

Figure 11. Approximation of the roller pin as a cantilever beam

The displacement $v_{L}$ at the free end of the pin turns out to be

$$
v_{L}=\sqrt{v_{x}^{2}+v_{y}^{2}}=\frac{F L^{3}}{3 E I}
$$

where $E$ is the Young modulus and $I=\pi a_{5}^{4} / 4$, with $a_{5}$ denoting the radius of the pin, is the polar moment of inertia of the cross section. Moreover, $v_{x}$ and $v_{y}$ are the pin elastic displacements in the $x$ - and $y$-directions, respectively, at the free end.

Before proceeding, we prove that the vertical component $f_{y}$ of the transmitted force is constant, and hence, we will consider only the magnitude of the $x$-component of $v_{L}$. Since we assume that the mechanism undergoes a pure-rolling motion, the force exerted by the cam onto the roller, denoted by $\mathbf{f}=\left[\begin{array}{ll}f_{x} & f_{y}\end{array}\right]^{T}$, passes through the center of the roller, i.e., its line of action passes through points $\mathrm{O}_{2}$ and $\mathrm{C}$, as depicted in Fig. 3. With a constant torque $\tau$ provided by the motor, we have $\tau=\| \mathbf{f}|| d$, where $d$ denotes the distance from the center of the input axis to the line of action of the force $\mathbf{f}$. Moreover, we have $d=b_{2} \sin \delta$. Hence, $\quad \tau=\|\mathbf{f}\|\left(b_{2} \sin \delta\right)=b_{2}(\|\mathbf{f}\| \sin \delta)=b_{2} f_{y}$. Finally, since $b_{2}=2 \pi / p$ we obtain the expression for $f_{y}$ sought:

$$
f_{y}=\frac{2 \pi \tau}{p}=F_{0}
$$

Since $\tau$ is constant, $f_{y}$ is also constant throughout one cycle. Consequently we only have to consider the $x$-component of $\mathbf{f}$, and hence, $v_{x}$ for the minimization problem:

$$
v_{x}=\frac{\left|f_{x}\right| L^{3}}{3 E I}=\frac{4\left|f_{x}\right| L^{3}}{3 E \pi a_{5}^{4}}=\beta \frac{\left|f_{x}\right|}{a_{5}^{4}}, \quad \beta=\frac{4 L^{3}}{3 E \pi}
$$

$\beta$ thus being a constant factor. The objective function $z$, to be minimized, is thus defined as

$$
z=\frac{f_{\max }^{2}}{a_{5}^{4}} \rightarrow \min _{\eta, a_{4}, a_{5}}
$$

where $f_{\max }$ is the maximum value of $f_{x}$ throughout one cycle. Since

$$
f_{x}=\frac{f_{y}}{\tan \delta}=\frac{F_{0}}{\tan \delta}
$$

we obtain

$$
z=\frac{1}{a_{5}^{4}} \max _{\psi}\left\{f_{x}^{2}\right\}=\frac{1}{a_{5}^{4}} \max _{\psi}\left\{\frac{F_{0}^{2}}{\tan ^{2} \delta}\right\}=\frac{F_{0}^{2}}{a_{5}^{4}} \max _{\psi}\left\{\frac{1}{\tan ^{2} \delta}\right\}
$$

with $\delta$, a function of $\psi$, given in Eq. (7c). Moreover, the system operates by means of two conjugate mechanisms, which alternately take over the power transmission. We established in Eq. (15) that when one mechanism is in positive action, $\psi$ is bounded between $\psi_{i}=\pi-\Delta$ and $\psi_{f}=2 \pi-\Delta$, which corresponds to $\delta$ bounded between $\delta_{i}$ and $\delta_{f}$ with $0 \leq \delta_{i}<\delta_{f} \leq \pi$. Moreover, functions $1 / \tan ^{2} \delta$ and $\cos ^{2} \delta$ are both unimodal in $-\pi \leq \delta \leq 0$ and in $0 \leq \delta \leq \pi$, their common maxima finding themselves at $-\pi, 0$ and $\pi$. Since $\cos ^{2} \delta$ is better behaved than $1 / \tan ^{2} \delta$, we redefine $z$ as

$$
z=\frac{1}{a_{5}^{4}} \max _{\delta_{i} \leq \delta \leq \delta_{f}}\left\{\cos ^{2} \delta\right\}
$$

Copyright (C) 2004 by ASME 
Furthermore, the function $\cos ^{2} \delta$ attains its global minimum of 0 in $[0, \pi]$, its maximum in the interval $\left[\delta_{i}, \delta_{f}\right]$, included in $[0, \pi]$, occurring at the larger of the two extremes of the interval, $\delta_{i}$ or $\delta_{f}$. It follows that the objective function to be minimized becomes

$$
z=\frac{1}{a_{5}^{4}} \max \left\{\cos ^{2} \delta_{i}, \cos ^{2} \delta_{f}\right\}
$$

with $\delta_{i}$ and $\delta_{f}$ the values of $\delta$ for $\psi_{i}=\pi-\Delta$ and $\psi_{f}=2 \pi-\Delta$, respectively. Using the expression for $\delta$ given in Eq. (77 ) and the trigonometric identity

$$
\cos (\arctan x)=\frac{1}{\sqrt{1+x^{2}}}
$$

we obtain the expression for $\cos ^{2} \delta$ :

$$
\cos ^{2} \delta=\frac{(2 \pi \eta-1)^{2}}{(2 \pi \eta-1)^{2}+(\psi-\pi)^{2}}
$$

Hence,

$$
\begin{aligned}
\cos ^{2} \delta_{i} & =\frac{(2 \pi \eta-1)^{2}}{(2 \pi \eta-1)^{2}+\left(\psi_{i}-\pi\right)^{2}} \\
\cos ^{2} \delta_{f} & =\frac{(2 \pi \eta-1)^{2}}{(2 \pi \eta-1)^{2}+\left(\psi_{f}-\pi\right)^{2}}
\end{aligned}
$$

Furthermore, since $\psi_{i}=\pi-\Delta, \psi_{f}=2 \pi-\Delta$ and $\Delta<0$, we have $\psi_{f}>\psi_{i}>\pi, \psi_{f}-\pi>\psi_{i}-\pi>0$ and, consequently, from Eqs. (36a $\&$ b), $\cos ^{2} \delta_{i}>\cos ^{2} \delta_{f}$ and the objective function to minimize becomes

$$
z=\frac{\cos ^{2} \delta_{i}}{a_{5}^{4}} \rightarrow \min _{\eta, a_{4}, a_{5}}
$$

with $\cos ^{2} \delta_{i}$ given in Eq. (36a) and $\psi_{i}=\pi-\Delta$.

\subsection{Geometric Constraints}

Two neighboring pins cannot be tangent to each other, as depicted in Fig. 12, and hence the radius $a_{5}$ of the pin is bounded as

$$
a_{5} / p<1 / 4
$$

Furthermore, $a_{4}$ and $a_{5}$ are not independent. From the SKF catalogue, for example, we have information on bearings available

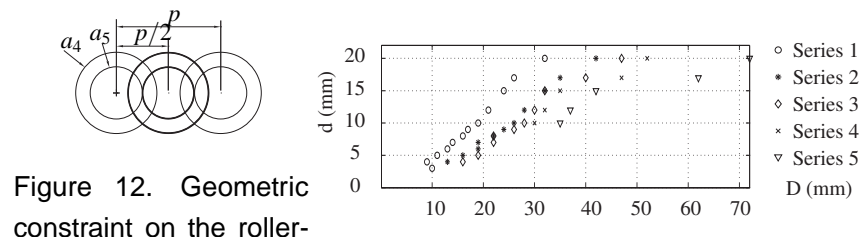

constraint on the roller-

pin radius

Figure 13. Dimensions of the SKF bearings

in terms of the outer radius $D$ and the inner radius $d$, as shown in Fig. 13. We divide these bearings into five series, from 1 to 5. Hence, each series can be represented by a continuous function. We chose series 2 , in which the basic dynamic load rating $C$ lies between 844 and 7020 N. Furthermore, for series 2, the relation between $D$ and $d$ can be approximated by a linear function $D$ vs. $d, D \simeq 1.6 d+10$ (in mm). Since $D=2 a_{4}$ and $d=2 a_{5}$, the above equation leads to

$$
a_{4} \simeq 1.6 a_{5}+5 \text { in } \mathrm{mm}
$$

We define now two non-dimensional parameters $\alpha_{4}$ and $\alpha_{5}$ :

$$
\alpha_{4}=a_{4} / p \quad \alpha_{5}=a_{5} / p
$$

where $\alpha_{5}$ can be derived from Eq. (39) as

$$
\alpha_{5}=(5 / 8) \alpha_{4}-25 /(8 p) \quad \text { with } p \text { in } \mathrm{mm} \text {. }
$$

The optimization problem is now expressed as

$$
z\left(\eta, \alpha_{4}\right)=\frac{\cos ^{2} \delta_{i}}{\alpha_{5}^{4}} \rightarrow \min _{\eta, \alpha_{4}}
$$

Moreover, we recall the geometric constraint defined in Eqs. (23) and (29) that can be rewritten as

$$
\begin{aligned}
& g_{1}=\frac{1}{\pi}-\eta \leq 0 \\
& g_{2}=\alpha_{4}-\frac{1}{2}<0 \\
& g_{3}=\alpha_{4}-\frac{1}{p \kappa_{p \max }}<0 \\
& g_{4}=\alpha_{4}-\eta+\frac{b}{p} \leq 0 \\
& g_{5}=\alpha_{5}-\frac{1}{4}<0
\end{aligned}
$$

with $\cos ^{2} \delta_{i}$ and $\alpha_{5}$ given in Eqs. (36a) and (41), respectively. 


\subsection{Results of the Optimization Problem}

We solve the foregoing optimization problem with an algorithm implemented Matlab, using $p=50 \mathrm{~mm}$ and $b=9.5 \mathrm{~mm}$. One solution is found, corresponding to $\eta=0.69$ and $a_{4}=$ $24.9992 \mathrm{~mm}$, with a value of $z=249$. The algorithm finds the values of $\eta$ and $a_{4}$ as big as possible considering the constraints. For this solution, constraints (43) $\&$ e) are active. Nevertheless, as we saw in subsection 3.3. about the influence of parameters $h$ and $a_{4}$, we want these parameters to be as small as possible in order to have low pressure-angle values. For the solution found above, the service factor is equal to $0 \%$. Consequently, we must find a compromise between the pressure angle and the roller-pin elastic deformation. Table 1 shows solutions found by the optimization algorithm upon reducing the boundaries of $\eta$. Each time the algorithm finds the corresponding value of $a_{4}$ as big as possible, constraint (43d) becomes active. Recorded in this table is also the corresponding maximum elastic deformation of the roller pin $v_{L \max }$ (its expression is derived below), the minimum and the maximum absolute values of the pressure angle, $\left|\mu_{\min }\right|$ and $\left|\mu_{\max }\right|$, respectively, and the service factor, as defined in section 2.4. From Eqs. (30) and 32) we have

$$
v_{L}=\frac{\beta}{a_{5}^{4}} \sqrt{f_{x}^{2}+f_{y}^{2}}
$$

Using Eqs. (31) and (34), the above equation leads to

$$
v_{L}=\frac{\beta F_{0}}{a_{5}^{4}} \sqrt{1+\frac{1}{\tan ^{2} \delta}}
$$

Which can be simplified by means of the expression for $\cos ^{2} \delta_{i}$ given in Eq. 36a as

$$
v_{L \max }=\frac{\beta F_{0}}{a_{5}^{4}} \frac{\sqrt{(2 \pi \eta-1)^{2}+\left(\psi_{i}-\pi\right)^{2}}}{\left|\psi_{i}-\pi\right|}
$$

with $\beta, F_{0}$ and $a_{5}$ given in Eqs. (32), (31) and (39), respectively, and $\psi_{i}=\pi-\Delta$. In Table 11 we record the value of $v_{L \max }$ with $L=10 \mathrm{~mm}, \tau=1.2 \mathrm{Nm}$ (according to the Orthoglide specifications recalled in section 1) and $E=2 \times 10^{5} \mathrm{MPa}$. We conclude from Table 1 that for this cam profile we cannot find an acceptable compromise between a low deformation of the roller pin, and hence a high stiffness and accuracy of the mechanism, and low pressure-angle values. Indeed, for an acceptable deformation of the roller pin, $v_{L \max }=8.87 \mu \mathrm{m}$, obtained with $\eta=0.38$, the service factor equals $54.68 \%$, which is too low. On the other hand, for an acceptable service factor of $79.43 \%$, obtained with $\eta=1 / \pi$, the deformation of the roller pin is equal to $710.19 \mu \mathrm{m}$.

\begin{tabular}{|c|l|l|l|l|l|l|l|}
\hline$\eta$ & $\begin{array}{l}a_{4} \\
(\mathrm{~mm})\end{array}$ & $\begin{array}{l}a_{5} \\
(\mathrm{~mm})\end{array}$ & $z$ & $\begin{array}{l}v_{L \max } \\
(\mu \mathrm{m})\end{array}$ & $\begin{array}{l}\left|\mu_{\min }\right| \\
\left({ }^{\circ}\right)\end{array}$ & $\begin{array}{l}\left|\mu_{\max }\right| \\
\left(^{\circ}\right)\end{array}$ & $\begin{array}{l}\text { service } \\
\text { factor }(\%)\end{array}$ \\
\hline 0.69 & 24.99 & 12.50 & 249 & 0.09 & 42.11 & 80.68 & 0 \\
0.5 & 15.5 & 6.56 & 2968 & 0.50 & 28.59 & 69.81 & 6.85 \\
0.4 & 10.5 & 3.44 & 32183 & 4.32 & 20.31 & 57.99 & 46.68 \\
0.39 & 10 & 3.12 & 45490 & 6.07 & 19.46 & 56.42 & 50.68 \\
0.38 & 9.5 & 2.81 & 66659 & 8.87 & 18.61 & 54.78 & 54.68 \\
0.37 & 9 & 2.50 & 102171 & 13.63 & 17.75 & 53.04 & 58.69 \\
0.36 & 8.5 & 2.19 & 165896 & 22.31 & 16.89 & 51.22 & 62.69 \\
0.35 & 8 & 1.87 & 290765 & 39.71 & 16.03 & 49.31 & 66.70 \\
0.34 & 7.5 & 1.56 & 566521 & 79.18 & 15.17 & 47.31 & 70.72 \\
0.33 & 7 & 1.25 & $1.2910^{6}$ & 186.06 & 14.31 & 45.21 & 74.73 \\
$1 / \pi$ & 6.41 & 0.88 & $4.6810^{6}$ & 710.19 & 13.31 & 42.64 & 79.43 \\
\hline
\end{tabular}

Table 1. Results of the optimization problem, with $p=50 \mathrm{~mm}, b=$ $9.5 \mathrm{~mm}, L=10 \mathrm{~mm}, \tau=1.2 \mathrm{Nm}$ and $E=2 \times 10^{5} \mathrm{MPa}$

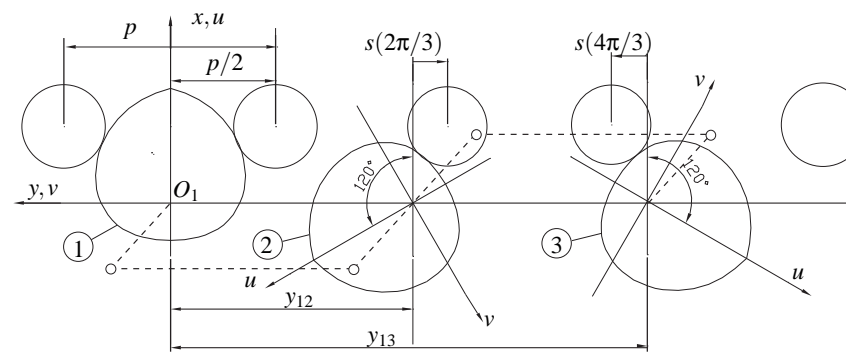

Figure 14. Layout of the non-coaxial conjugate-cam mechanism

\section{A Non-Coaxial Conjugate-Cam Mechanism}

This Section describes a new mechanism, based on SlideO-Cam, that enables us to decrease considerably the pressure angle while meeting the Orthoglide specifications. This mechanism is composed of three conjugate cams mounted on three parallel shafts, the rollers being placed on one single side of the roller-carrying slider. One motor provides the torque to the central camshaft, this torque then being transmitted to the two other camshafts through a parallelogram mechanism coupling them, whose detailed design is reported in [10]. We denote by 1,2 and 3 the three cams, as shown in Fig. 14. The profile of each cam is described in Section 2. The cams are mounted in such a way that the angle between the $u$-axis of cam 1 and cam 2 is $120^{\circ}$, and the angle between the $u$-axis of cam 1 and cam 3 is $240^{\circ}$. According to the configuration of the mechanism depicted in Fig. 14, and denoting by $y_{12}$ and $y_{13}$ the distance between the origin of 1 and 2 , and between the origin of 1 and 3, respectively, we have

$$
y_{12}=p / 2+p+s(2 \pi / 3), \quad y_{13}=p / 2+2 p+s(4 \pi / 3)
$$

Using the expression of the input-output function $s$ given in Eq. (11), we obtain

$$
y_{12}=4 p / 3, \quad y_{13}=8 p / 3
$$


Figure 15 shows the pressure angle variation for each cam vs. $\psi$ where 1,2 and 3 denote the plot of the pressure angle for cams 1,2 and 3 , respectively. Moreover, cams 2 and 3 are rotated by angles $2 \pi / 3$ and $4 \pi / 3$, respectively, from cam 1 . We can also consider that the plot for cam 3 that drives the follower before cam 1 in a previous cycle, refereed to as 3 ' in Fig. 15, is a translation of $-2 \pi / 3$ from cam 1 . As we saw in Eq. (14), cam 1 can drive the follower within $\pi \leq \psi \leq 2 \pi-\Delta$, which corresponds in Fig. 15 to the part of the plot 1 between points $B$ and $D$. Consequently, cam 2 can drive the follower within

$\pi+2 \pi / 3 \leq \psi \leq 2 \pi-\Delta+2 \pi / 3$ i.e. $5 \pi / 3 \leq \psi \leq 8 \pi / 3-\Delta$

and cam 3 within

$\pi+4 \pi / 3 \leq \psi \leq 2 \pi-\Delta+4 \pi / 3$ i.e. $7 \pi / 3 \leq \psi \leq 10 \pi / 3-\Delta$

which is equivalent to saying that cam 3 can drive the follower in a previous cycle, within

$$
\pi-2 \pi / 3 \leq \psi \leq 2 \pi-\Delta-2 \pi / 3 \text { i.e. } \pi / 3 \leq \psi \leq 4 \pi / 3-\Delta
$$

The above interval corresponds in Fig. 15 to the part of the plot 3 ' between points $A$ and $C$. Consequently, there is a common part for cams 1 (plot 1 ) and 3 (plot 3 ') during which these two cams can drive the follower, namely, between points $B$ and $C$, which corresponds to $\pi \leq \psi \leq 4 \pi / 3-\Delta$. Moreover, during this common part, cam 3 has lower absolute pressure angle values than 1, and hence, we consider that only cam 3 drives the follower. Consequently, cam 1 drives the follower only within $4 \pi / 3-\Delta \leq \psi \leq 2 \pi-\Delta$. These boundaries allow us to have a pressure angle lower than with coaxial conjugate cams, since we do not use anymore the part of the cam profile within $\pi-\Delta \leq \psi \leq 4 \pi / 3-\Delta$, which has high absolute pressure angle values. We can thus obtain a higher service factor for the mechanism. The drive thus design is sketched in Fig. 16.
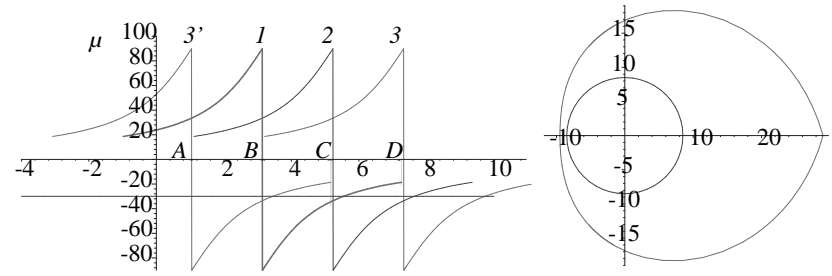

Figure 15. Pressure angle for the three Figure 16. A sketch of the cams prismatic drive

\section{Conclusions}

The non-coaxial conjugate-cam mechanism reported here allows us to drive the Orthoglide with prismatic actuators using rotary DC motors. Moreover, the maximum roller-pin deformation $v_{L \max }$ derived in eq. (44) is reasonable low. In Table 22, we

\begin{tabular}{|l|l|l|l|l|l|l|}
\hline$\eta$ & $a_{4}(\mathrm{~mm})$ & $a_{5}(\mathrm{~mm})$ & $\begin{array}{l}v_{L \max } \\
(\mu \mathrm{m})\end{array}$ & $\left|\mu_{\min }\right|\left(^{\circ}\right)$ & $\left|\mu_{\max }\right|\left(^{\circ}\right)$ & $\begin{array}{l}\text { service } \\
\text { factor }(\%)\end{array}$ \\
\hline 0.5 & 15.5 & 6.56 & 0.26 & 28.59 & 49.41 & 10.49 \\
0.4 & 10.5 & 3.44 & 2.88 & 20.31 & 37.20 & 70.02 \\
0.39 & 10 & 3.12 & 4.14 & 19.46 & 35.81 & 76.02 \\
0.38 & 9.5 & 2.81 & 6.20 & 18.61 & 34.39 & 82.02 \\
0.37 & 9 & 2.50 & 9.76 & 17.75 & 32.95 & 88.03 \\
0.36 & 8.5 & 2.19 & 16.39 & 16.89 & 31.48 & 94.04 \\
0.35 & 8 & 1.87 & 29.89 & 16.03 & 29.98 & 100 \\
0.34 & 7.5 & 1.56 & 61.07 & 15.17 & 28.47 & 100 \\
0.33 & 7 & 1.25 & 147.02 & 14.31 & 26.93 & 100 \\
$1 / \pi$ & 6.41 & 0.88 & 576.95 & 13.31 & 25.12 & 100 \\
\hline
\end{tabular}

Table 2. Design parameters, roller pin deformation and pressure angle for the non-coaxial conjugate-cam mechanism, with $p=50 \mathrm{~mm}, b=$ $9.5 \mathrm{~mm}, L=10 \mathrm{~mm}, \tau=1.2 \mathrm{Nm}$ and $E=2 \times 10^{5} \mathrm{MPa}$.

record the values of $\eta, a_{4}, a_{5}, v_{L \max },\left|\mu_{\min }\right|,\left|\mu_{\max }\right|$ and the service factor for the non-coaxial conjugate-cam mechanism. The best compromise is to use the non-coaxial conjugate-cam mechanism with $\eta=0.37$, whence the radius of the roller is $a_{4}=9 \mathrm{~mm}$ and the roller-pin deformation is $v_{L \max }=9.76 \mu \mathrm{m}$ with a good service factor of $88.03 \%$.

\section{REFERENCES}

[1] González-Palacios, M. A. and Angeles, J., 2000, "The novel design of a pure-rolling transmission to convert rotational into translational motion," Proc. 2000 ASME Design Engineering Technical Conferences, Baltimore, Sept. 10-13, CD-ROM.

[2] Chablat, D. and Wenger Ph. 2003, "Architecture Optimization of a 3-DOF Parallel Mechanism for Machining Applications, the Orthoglide," IEEE Transactions on Robotics and Automation, Vol. 19/3, pp. 403-410, June.

[3] Lampinen, J., 2003, "Cam shape optimisation by genetic algorithm," Computer-Aided Design, Vol. 35, pp. 727-737.

[4] Bouzakis, K.D., Mitsi, S. and Tsiafis, J., 1997, "Computer-Aided Optimum Design and NC Milling of Planar Cam Mechanims," Int. J. Math. Tools Manufaet. Vol. 37, No. 8, pp. 1131-1142.

[5] Gonzáles-Palacios, M. A. and Angeles, J., 1993, Cam Synthesis, Kluwer Academic Publishers B.V., Dordrecht.

[6] Waldron, K. J. and Kinzel, G. L., 1999, Kinematics, Dynamics, and Design of Machinery, John Wiley \& Sons, Inc., New York.

[7] Lee, M.K., 2001, Design for Manufacturability of Speed-Reduction Cam Mechanisms, M.Eng. Thesis, Dept. of Mechanical Engineering, McGill University, Montreal.

[8] Angeles, J. and López-Cajún,C., 1991, Optimization of Cam Mechanisms, Kluwer Academic Publishers B.V., Dordrecht.

[9] Teng, C.P. and Angeles, J., 2004, "An optimality criterion for the structural optimization of machine element," ASME Journal of Mechanical Design, Accepted for publication.

[10] Renotte, J. and Angeles, J. "The Design of a Pure-Rolling Ca Mechanism to Convert Rotationnal into Translational Motion," Internal Report, TR-CIM-03-06, August, 2003. 